\title{
Near-Optimal Nash Strategy for Multiparameter Singularly Perturbed Systems
}

\author{
Hiroaki Mukaidani and Hua Xu
}

\begin{abstract}
In this paper, the linear quadratic Nash games for infinite horizon multiparameter singularly perturbed systems (MSPS) are discussed. The uniqueness and the asymptotic structure of the solution to the generalized cross-coupled multiparameter algebraic Riccati equations (GCMARE) are newly established without the nonsingularity assumptions for the fast state matrices. The main contribution of this paper is that a construction of high-order approximations to a strategy that guarantees a desired performance level on the basis of a new iterative technique is proposed. As a result, it is shown that the high-order accuracy strategy improves the performance.
\end{abstract}

\section{INTRODUCTION}

The control problems for the multiparameter singularly perturbed systems (MSPS) have been investigated extensively (see e.g., [1] and reference therein). In these various studies of the MSPS, the linear quadratic Nash games for MSPS have been studied [2], [3]. Recent advance in theory of numerical computation for the singularly perturbed systems (SPS) and the MSPS has allowed a revisiting of Nash games [4], [7], [8]. The numerical method is a very powerful tool, it can not only efficiently find feasible solutions, but also easily handle reduced-order calculation. However, a limitation of these approaches is that the small parameters are assumed to be known. Thus, it is not applicable to a large class of problems where the parameters represent small unknown perturbations whose values are not known exactly.

It is well-known that one of the approaches for constructing Nash equilibrium strategies of the MSPS is the composite design method [2], [3]. When the parameters represent the small unknown perturbations, the composite strategies are very useful. However, the composite Nash equilibrium solution achieves only a performance which is $O(\mu)$ (where $\mu:=\sqrt{\varepsilon_{1} \varepsilon_{2}}$ ) close to the full-order performance for small enough $\mu$. Therefore, for values of $\mu$ that are not too small, higher order approximations based on the reduced-order equations are needed to guarantee the desired performance.

In this paper, the linear quadratic Nash games for infinite horizon MSPS are studied. We first investigate the uniqueness and boundedness of the solution to the generalized cross-coupled multiparameter algebraic Riccati equations

This work was supported in part by the Electric Technology Research Foundation of Chugoku of Japan.

H. Mukaidani is with Graduate School of Education, Hiroshima University, 1-1-1 Kagamiyama, Higashi-Hiroshima, 739-8524 Japan. mukaida@hiroshima-u.ac.jp

H. Xu is with Graduate School of Business Sciences, The University of Tsukuba, 3-29-1, Otsuka, Bunkyo-ku, Tokyo, 112-0012 Japan. xuhua@gssm.otsuka.tsukuba.ac.jp
(GCMARE) and newly establish its asymptotic structure without nonsingularity assumption that the fast state matrices are invertiable. The main contribution of this paper is to propose the high-order approximate strategy via a new iterative method. This leads to effective asymptotic and numerical algorithm. It is worth pointing out that the proposed high-order approximate strategy can be constructed by using of the approximate values of the small parameters. Numerical example shows that the proposed high-order accuracy strategy improves the performance compared with the existing parameter independent strategy.

Notation: The notations used in this paper are fairly standard. The superscript $T$ denotes matrix transpose. $I_{n}$ denotes the $n \times n$ identity matrix. $E[\cdot]$ denotes the expection operator.

\section{PROBLEM FORMULATION}

Consider a linear time-invariant MSPS [1]

$$
\begin{aligned}
\dot{x}_{0} & =\sum_{i=0}^{2} A_{0 i} x_{i}+\sum_{i=1}^{2} B_{0 i} u_{i}, x_{0}(0)=x_{0}^{0}, \\
\varepsilon_{1} \dot{x}_{1} & =A_{10} x_{0}+A_{11} x_{1}+B_{11} u_{1}, x_{1}(0)=x_{1}^{0}, \\
\varepsilon_{2} \dot{x}_{2} & =A_{20} x_{0}+A_{22} x_{2}+B_{22} u_{2}, x_{2}(0)=x_{2}^{0}
\end{aligned}
$$

with quadratic cost functions

$$
\begin{aligned}
J_{i}\left(u_{i}, u_{j}\right)= & \frac{1}{2} \int_{0}^{\infty}\left[y_{i}^{T} y_{i}+u_{i}^{T} R_{i i} u_{i}+\mu u_{j}^{T} R_{i j} u_{j}\right] d t, \\
& R_{i i}>0, R_{i j} \geq 0, \mu:=\sqrt{\varepsilon_{1} \varepsilon_{2}}, \quad \text { (2a) } \\
y_{i}= & C_{i 0} x_{0}+C_{i i} x_{i}=C_{i} x, \\
x(t)= & {\left[\begin{array}{l}
x_{0}(t) \\
x_{1}(t) \\
x_{2}(t)
\end{array}\right], \quad i=1,2, }
\end{aligned}
$$

where $x_{i} \in \mathbf{R}^{n_{i}}, i=0,1,2$ are the state vectors and $u_{i} \in \mathbf{R}^{m_{i}}, i=1,2$ are the control inputs. All the matrices are constant matrices of appropriate dimensions.

$\varepsilon_{1}$ and $\varepsilon_{2}$ are two small positive singular bounded perturbation parameters of the same order of magnitude which are constrained by the known parameter $\sigma_{j}$ such that

$$
\begin{aligned}
& \bar{\varepsilon}_{j}-\sigma_{j} \bar{\mu}^{\eta} \leq \varepsilon_{j} \leq \bar{\varepsilon}_{j}+\sigma_{j} \bar{\mu}^{\eta}, j=1,2, \\
& 0<k_{1} \leq \alpha \equiv \frac{\varepsilon_{1}}{\varepsilon_{2}} \leq k_{2}<\infty,
\end{aligned}
$$

where $\bar{\mu}:=\sqrt{\bar{\varepsilon}_{1} \bar{\varepsilon}_{2}}, \bar{\varepsilon}_{j}$ and $\sigma_{j}, j=1,2$ are known constants. $\eta$ is some constant which has an appropriate 
degree of accuracy for parameter $\varepsilon_{j}$. It should be noted that the parameter $\varepsilon_{j}$ is unknown but its bound are known.

It is assumed that the limit of $\alpha$ exists as $\varepsilon_{1}$ and $\varepsilon_{2}$ tend to zero (see e.g., [1]), that is

$$
\bar{\alpha}=\lim _{\substack{\varepsilon_{1} \rightarrow+0 \\ \varepsilon_{2} \rightarrow+0}} \alpha .
$$

It is worth pointing out that the matrices $A_{i i}, i=1,2$ may be singular. In fact such systems arise in some real physical applications like a flexible space structure [6]. In this case, it should be noted that the composite design [2], [3] cannot be applied.

Let us introduce the partitioned matrices

$$
\begin{aligned}
& A:=\left[\begin{array}{ccc}
A_{00} & A_{01} & A_{02} \\
A_{10} & A_{11} & 0 \\
A_{20} & 0 & A_{22}
\end{array}\right], \\
& B_{1}:=\left[\begin{array}{c}
B_{01} \\
B_{11} \\
0
\end{array}\right], B_{2}:=\left[\begin{array}{c}
B_{02} \\
0 \\
B_{22}
\end{array}\right] \text {, } \\
& S_{1}:=B_{1} R_{11}^{-1} B_{1}^{T}=\left[\begin{array}{ccc}
S_{001} & S_{011} & 0 \\
S_{011}^{T} & S_{111} & 0 \\
0 & 0 & 0
\end{array}\right] \text {, } \\
& S_{2}:=B_{2} R_{22}^{-1} B_{2}^{T}=\left[\begin{array}{ccc}
S_{002} & 0 & S_{022} \\
0 & 0 & 0 \\
S_{022}^{T} & 0 & S_{222}
\end{array}\right] \text {, } \\
& G_{1}^{\mu}=\mu B_{1} R_{11}^{-1} R_{21} R_{11}^{-1} B_{1}^{T}=\left[\begin{array}{ccc}
G_{001}^{\mu} & G_{011}^{\mu} & 0 \\
G_{011}^{\mu T} & G_{111}^{\mu} & 0 \\
0 & 0 & 0
\end{array}\right] \text {, } \\
& G_{2}^{\mu}=\mu B_{2} R_{22}^{-1} R_{12} R_{22}^{-1} B_{2}^{T}=\left[\begin{array}{ccc}
G_{002}^{\mu} & 0 & G_{022}^{\mu} \\
0 & 0 & 0 \\
G_{022}^{\mu T} & 0 & G_{222}^{\mu}
\end{array}\right] \text {, } \\
& Q_{1}:=C_{1}^{T} C_{1}=\left[\begin{array}{ccc}
Q_{001} & Q_{011} & 0 \\
Q_{011}^{T} & Q_{111} & 0 \\
0 & 0 & 0
\end{array}\right] \text {, } \\
& Q_{2}:=C_{2}^{T} C_{2}=\left[\begin{array}{ccc}
Q_{002} & 0 & Q_{022} \\
0 & 0 & 0 \\
Q_{022}^{T} & 0 & Q_{222}
\end{array}\right] \text {. }
\end{aligned}
$$

We now consider the linear quadratic Nash games for infinite horizon nonstandard MSPS (1) under the following basic assumptions (see e.g., [2], [5]).

Assumption 1: There exists a $\mu^{*}>0$ such that the triples $\left(A_{e}, B_{i e}, C_{i}\right), i=1,2$ are stabilizable and detectable for all $\mu \in\left(0, \mu^{*}\right]$, where $\mu=\sqrt{\varepsilon_{1} \varepsilon_{2}}$.

Assumption 2: The triples $\left(A_{i i}, B_{i i}, C_{i i}\right), i=1,2$ are stabilizable and detectable.

These conditions are quite natural since at least one control agent has to be able to control and observe unstable modes. Our purpose is to find a linear feedback controller $\left(u_{1}^{*}, u_{2}^{*}\right)$ such that

$$
J_{i}\left(u_{i}^{*}, u_{j}^{*}\right) \leq J_{i}\left(u_{i}, u_{j}^{*}\right), \quad i, j=1,2, \quad i \neq j .
$$

Nash inequality shows that $u_{i}^{*}$ regulates the state to zero with minimum output energy. The following lemma is already known [2], [3].
Lemma 1: Under Assumption 1, there exists an admissible controller such that the inequality (5) holds iff the following full-order GCMARE

$$
\begin{gathered}
A^{T} X+X^{T} A+Q_{1}-X^{T} S_{1} X \\
-X^{T} S_{2} Y-Y^{T} S_{2} X+Y^{T} G_{2}^{\mu} Y=0 \\
A^{T} Y+Y^{T} A+Q_{2}-Y^{T} S_{2} Y \\
-Y^{T} S_{1} X-X^{T} S_{1} Y+X^{T} G_{1}^{\mu} X=0
\end{gathered}
$$

have solutions $\Phi_{e} X \geq 0$ and $\Phi_{e} Y \geq 0$, where

$$
\begin{aligned}
X:= & {\left[\begin{array}{ccc}
X_{00} & \varepsilon_{1} X_{10}^{T} & \varepsilon_{2} X_{20}^{T} \\
X_{10} & X_{11} & \sqrt{\alpha}^{-1} X_{21}^{T} \\
X_{20} & \sqrt{\alpha} X_{21} & X_{22}
\end{array}\right], } \\
Y:= & {\left[\begin{array}{ccc}
Y_{00} & \varepsilon_{1} Y_{10}^{T} & \varepsilon_{2} Y_{20}^{T} \\
Y_{10} & Y_{11} & \sqrt{\alpha}^{-1} Y_{21}^{T} \\
Y_{20} & \sqrt{\alpha} Y_{21} & Y_{22}
\end{array}\right], } \\
\Phi_{e}:= & {\left[\begin{array}{ccc}
I_{n_{0}} & 0 & 0 \\
0 & \varepsilon_{1} I_{n_{1}} & 0 \\
0 & 0 & \varepsilon_{2} I_{n_{2}}
\end{array}\right] . }
\end{aligned}
$$

Then the closed-loop linear Nash equilibrium solutions to the full-order problem are given by

$$
\begin{aligned}
& u_{1}^{*}(t)=-R_{11}^{-1} B_{1}^{T} X x(t), \\
& u_{2}^{*}(t)=-R_{22}^{-1} B_{2}^{T} Y x(t) .
\end{aligned}
$$

It should be noted that it is impossible to solve the GCMARE (6) because the small perturbed parameter $\varepsilon_{i}$ are partially unknown.

\section{ASYMPTOTIC STRUCTURE}

Nash equilibrium strategies for the MSPS will be studied under the following basic assumption, so that we can apply the proposed method to the nonstandard MSPS.

Assumption 3: The Hamiltonian matrices $T_{i i i}, i=1,2$ are nonsingular, where

$$
T_{i i i}:=\left[\begin{array}{cc}
A_{i i} & -S_{i i i} \\
-Q_{i i i} & -A_{i i}^{T}
\end{array}\right] .
$$

Under Assumptions 2 and 3, using the similar technique in [9], we can obtain the following zeroth-order equations of the GCMARE (6) as $\mu \rightarrow+0$

$$
\begin{aligned}
& A_{s}^{T} \bar{X}_{00}+\bar{X}_{00} A_{s}+Q_{s 1}-\bar{X}_{00} S_{s 1} \bar{X}_{00} \\
& -\bar{X}_{00} S_{s 2} \bar{Y}_{00}-\bar{Y}_{00} S_{s 2} \bar{X}_{00}=0, \\
& A_{s}^{T} \bar{Y}_{00}+\bar{Y}_{00} A_{s}+Q_{s 2}-\bar{Y}_{00} S_{s 2} \bar{Y}_{00} \\
& -\bar{Y}_{00} S_{s 1} \bar{X}_{00}-\bar{X}_{00} S_{s 1} \bar{Y}_{00}=0, \\
& A_{11}^{T} \bar{X}_{11}+\bar{X}_{11} A_{11}-\bar{X}_{11} S_{111} \bar{X}_{11}+Q_{111}=0 \text {, } \\
& A_{22}^{T} \bar{Y}_{22}+\bar{Y}_{22} A_{22}-\bar{Y}_{22} S_{222} \bar{Y}_{22}+Q_{222}=0 \text {, } \\
& \bar{X}_{22}=0, \bar{Y}_{11}=0 \text {, } \\
& {\left[\begin{array}{c}
\bar{X}_{10}^{T} \\
\bar{Y}_{10}^{T}
\end{array}\right]^{T}=\left[\begin{array}{ll}
\bar{X}_{11} & -I_{n_{1}}
\end{array}\right] T_{111}^{-1} T_{101}\left[\begin{array}{cc}
I_{n_{0}} & 0 \\
\bar{X}_{00} & \bar{Y}_{00}
\end{array}\right]} \\
& {\left[\begin{array}{c}
\bar{X}_{20}^{T} \\
\bar{Y}_{20}^{T}
\end{array}\right]^{T}=\left[\begin{array}{ll}
\bar{Y}_{22} & -I_{n_{2}}
\end{array}\right] T_{222}^{-1} T_{202}\left[\begin{array}{cc}
0 & I_{n_{0}} \\
\bar{X}_{00} & \bar{Y}_{00}
\end{array}\right],}
\end{aligned}
$$


where

$$
\begin{aligned}
& {\left[\begin{array}{cc}
A_{s} & * \\
* & -A_{s}^{T}
\end{array}\right]} \\
& =\left[\begin{array}{cc}
A_{00} & \star \\
* & -A_{00}^{T}
\end{array}\right]-T_{011} T_{111}^{-1} T_{101}-T_{022} T_{222}^{-1} T_{202}, \\
& {\left[\begin{array}{cc}
* & -S_{s 1} \\
-Q_{s 1} & \star
\end{array}\right]=T_{001}-T_{011} T_{111}^{-1} T_{101},} \\
& \left.\quad \begin{array}{cc}
* & -S_{s 2} \\
-Q_{s 2} & *
\end{array}\right]=T_{002}-T_{022} T_{222}^{-1} T_{202}, \\
& T_{001}=\left[\begin{array}{cc}
A_{00} & -S_{001} \\
-Q_{001} & -A_{00}^{T}
\end{array}\right], T_{011}=\left[\begin{array}{cc}
A_{01} & -S_{011} \\
-Q_{011} & -A_{10}^{T}
\end{array}\right], \\
& T_{101}=\left[\begin{array}{cc}
A_{10} & -S_{011}^{T} \\
-Q_{011}^{T} & -A_{01}^{T}
\end{array}\right], T_{002}=\left[\begin{array}{cc}
A_{00} & -S_{002} \\
-Q_{002} & -A_{00}^{T}
\end{array}\right], \\
& T_{022}=\left[\begin{array}{cc}
A_{02} & -S_{022} \\
-Q_{022} & -A_{20}^{T}
\end{array}\right], T_{202}=\left[\begin{array}{cc}
A_{022} \\
-Q_{022}^{T} & -A_{02}^{T}
\end{array}\right] .
\end{aligned}
$$

The following theorem shows the relation between the solutions $X$ and $Y$ and the zeroth-order solutions $\bar{X}_{l m}$ and $\bar{Y}_{l m}, l m=00,10,20,11,21,22$.

Theorem 1: Suppose that

$$
\begin{gathered}
\operatorname{det} \Gamma\left[\begin{array}{c}
\hat{A}_{s}^{T} \otimes I_{n_{0}}+I_{n_{0}} \otimes \hat{A}_{s}^{T} \\
-\left[\left(S_{s 1} \bar{Y}_{00}\right)^{T} \otimes I_{n_{0}}+I_{n_{0}} \otimes\left(S_{s 1} \bar{Y}_{00}\right)^{T}\right] \\
-\left[\left(S_{s 2} \bar{X}_{00}\right)^{T} \otimes I_{n_{0}}+I_{n_{0}} \otimes\left(S_{s 2} \bar{X}_{00}\right)^{T}\right] \\
\hat{A}_{s}^{T} \otimes I_{n_{0}}+I_{n_{0}} \otimes \hat{A}_{s}^{T}
\end{array}\right]
\end{gathered}
$$

$\neq 0$

where $\hat{A}_{s}:=A_{s}-S_{s 1} \bar{X}_{00}-S_{s 2} \bar{Y}_{00}$ and the matrix $\hat{A}_{s}$ is stable.

Under Assumptions 1-3, the GCMARE (6) admits the solutions $X$ and $Y$ such that these matrices possess the power series expansion at $\mu=0$. That is,

$$
\begin{aligned}
& X=\bar{X}+O(\mu)=\left[\begin{array}{ccc}
\bar{X}_{00} & 0 & 0 \\
\bar{X}_{10} & \bar{X}_{11} & 0 \\
\bar{X}_{20} & 0 & 0
\end{array}\right]+O(\mu), \\
& Y=\bar{Y}+O(\mu)=\left[\begin{array}{ccc}
\bar{Y}_{00} & 0 & 0 \\
\bar{Y}_{10} & 0 & 0 \\
\bar{Y}_{20} & 0 & \bar{Y}_{22}
\end{array}\right]+O(\mu),
\end{aligned}
$$

where for any positive integer $l, \lim _{\mu \rightarrow+0} \frac{O\left(\mu^{l}\right)}{\mu^{l}}<\infty$.

Proof: Since the proof can be done by using the similar technique in [9], it is omitted.

It should be noted that there are no matrices $G_{i e}^{\mu}, i=$ 1, 2 in the CMARE (6) which has been considered in [9]. Therefore, our result is extension of the existing one. In addition, it is worth pointing out that the asymptotic structures of the solutions $X$ and $Y$ are established without the assumption that the matrices $A_{i i}, i=1,2$ are nonsingular compared with the existing result in [2].

\section{ITERATIVE TECHNIQUE}

The solutions of (11) can be changed as follows.

$$
\begin{aligned}
X & =\bar{X}+\mu E \\
& =\bar{X}+\mu\left[\begin{array}{ccc}
E_{00} & \sqrt{\alpha} X_{10}^{T} & \sqrt{\alpha}^{-1} X_{20}^{T} \\
E_{10} & E_{11} & \sqrt{\alpha}^{-1} E_{21}^{T} \\
E_{20} & \sqrt{\alpha} E_{21} & E_{22}
\end{array}\right], \\
Y & =\bar{Y}+\mu F \\
& =\bar{Y}+\mu\left[\begin{array}{ccc}
F_{00} & \sqrt{\alpha} Y_{10}^{T} & \sqrt{\alpha}^{-1} Y_{20}^{T} \\
F_{10} & F_{11} & \sqrt{\alpha}^{-1} F_{21}^{T} \\
F_{20} & \sqrt{\alpha} F_{21} & F_{22}
\end{array}\right],
\end{aligned}
$$

where $X_{j 0}=\bar{X}_{j 0}+\mu E_{j 0}$ and $Y_{j 0}=\bar{Y}_{j 0}+\mu F_{j 0}, j=1,2$.

Substituting (12) into the GCMARE (6) and using

$$
\begin{gathered}
A^{T} \bar{X}+\bar{X}^{T} A+Q_{1}-\bar{X}^{T} S_{1} \bar{X} \\
-\bar{X}^{T} S_{2} \bar{Y}-\bar{Y}^{T} S_{2} \bar{X}=0, \\
A^{T} \bar{Y}+\bar{Y}^{T} A+Q_{2}-\bar{Y}^{T} S_{2} \bar{Y} \\
-\bar{Y}^{T} S_{1} \bar{X}-\bar{X}^{T} S_{1} \bar{Y}=0
\end{gathered}
$$

we have

$$
\begin{gathered}
D^{T} E+E^{T} D-L^{T} F-F^{T} L+Y^{T} G_{2} Y \\
-\mu\left(E^{T} S_{1} E+E^{T} S_{2} F+F^{T} S_{2} E\right)=0 \\
D^{T} F+F^{T} D-M^{T} E-E^{T} M+X^{T} G_{1} X \\
-\mu\left(F^{T} S_{2} F+F^{T} S_{1} E+E^{T} S_{1} F\right)=0,
\end{gathered}
$$

where

$$
\begin{aligned}
& E=\left[\begin{array}{ccc}
E_{00} & \sqrt{\alpha} X_{10}^{T} & \sqrt{\alpha}^{-1} X_{20}^{T} \\
E_{10} & E_{11} & \sqrt{\alpha}^{-1} E_{21}^{T} \\
E_{20} & \sqrt{\alpha} E_{21} & E_{22}
\end{array}\right], \\
& F=\left[\begin{array}{ccc}
F_{00} & \sqrt{\alpha} Y_{10}^{T} & \sqrt{\alpha}^{-1} Y_{20}^{T} \\
F_{10} & F_{11} & \sqrt{\alpha}^{-1} F_{21}^{T} \\
F_{20} & \sqrt{\alpha} F_{21} & F_{22}
\end{array}\right] \text {, } \\
& D=A-S_{1} \bar{X}-S_{2} \bar{Y}=\left[\begin{array}{ccc}
D_{00} & D_{x 01} & D_{y 02} \\
D_{x 10} & D_{x 11} & 0 \\
D_{y 20} & 0 & D_{y 22}
\end{array}\right] \text {, } \\
& L=S_{2} \bar{X}=\left[\begin{array}{ccc}
L_{x 00} & 0 & 0 \\
0 & 0 & 0 \\
L_{x 20} & 0 & 0
\end{array}\right] \\
& M=S_{1} \bar{Y}=\left[\begin{array}{ccc}
M_{y 00} & 0 & 0 \\
M_{y 10} & 0 & 0 \\
0 & 0 & 0
\end{array}\right] \text {, } \\
& D_{x 01}=A_{01}-S_{011} \bar{X}_{11}, D_{x 11}=A_{11}-S_{111} \bar{X}_{11} \text {, } \\
& D_{y 02}=A_{02}-S_{022} \bar{Y}_{22}, D_{y 22}=A_{22}-S_{222} \bar{Y}_{22} \text {, } \\
& D_{00}=A_{00}-S_{001} \bar{X}_{00}-S_{002} \bar{Y}_{00}-S_{011} \bar{X}_{10}-S_{022} \bar{Y}_{20} \text {, } \\
& D_{x 10}=A_{10}-S_{011}^{T} \bar{X}_{00}-S_{111} \bar{X}_{10} \text {, } \\
& D_{y 20}=A_{20}-S_{022}^{T} \bar{Y}_{00}-S_{222} \bar{Y}_{20} \text {, } \\
& L_{x 00}=S_{002} \bar{X}_{00}+S_{022} \bar{X}_{20} \text {, } \\
& L_{x 20}=S_{022}^{T} \bar{X}_{00}+S_{222} \bar{X}_{20},
\end{aligned}
$$


$M_{y 00}=S_{001} \bar{Y}_{00}+S_{011} \bar{Y}_{10}$,

$M_{y 10}=S_{011}^{T} \bar{Y}_{00}+S_{111} \bar{Y}_{10}$,

$G_{1}=B_{1} R_{11}^{-1} R_{21} R_{11}^{-1} B_{1}^{T}, G_{2}=B_{2} R_{22}^{-1} R_{12} R_{22}^{-1} B_{2}^{T}$.

When the parameters $\varepsilon_{j}$ are known, the GCMARE (14) can be solved by using the existing results [7], [8]. However, the exact values are unknown for the considered problem in this paper. Hence, we propose the new following iterative algorithm (15) which does not depend on information of these parameters.

$$
\begin{aligned}
& D^{T} E^{(n+1)}+E^{(n+1) T} D-L^{T} F^{(n+1)}-F^{(n+1) T} L \\
& \quad+Y^{(n) T} G_{2} Y^{(n)}-\bar{\mu}\left(E^{(n) T} S_{1} E^{(n)}\right. \\
& \left.\quad+E^{(n) T} S_{2} F^{(n)}+F^{(n) T} S_{2} E^{(n)}\right)=0, \\
& D^{T} F^{(n+1)}+F^{(n+1) T} D-M^{T} E^{(n+1)}-E^{(n+1) T} M \\
& \quad+X^{(n) T} G_{1} X^{(n)}-\bar{\mu}\left(F^{(n) T} S_{2} F^{(n)}\right. \\
& \left.\quad+F^{(n) T} S_{1} E^{(n)}+E^{(n) T} S_{1} F^{(n)}\right)=0,
\end{aligned}
$$

where the integer of $n$ means the necessary iteration number to attain the desired performance level. $\tilde{\alpha}:=\frac{\bar{\varepsilon}_{1}}{\bar{\varepsilon}_{2}}, \bar{\mu}:=$ $\sqrt{\bar{\varepsilon}_{1} \bar{\varepsilon}_{2}}$,

$$
\begin{aligned}
E^{(n)} & =\left[\begin{array}{ccc}
E_{00}^{(n)} & \sqrt{\tilde{\alpha}} X_{10}^{(n) T} & \sqrt{\tilde{\alpha}}^{-1} X_{20}^{(n) T} \\
E_{10}^{(n)} & E_{11}^{(n)} & \sqrt{\tilde{\alpha}}^{-1} E_{21}^{(n) T} \\
E_{20}^{(n)} & \sqrt{\tilde{\alpha}} E_{21}^{(n)} & E_{22}^{(n)}
\end{array}\right], \\
F^{(n)} & =\left[\begin{array}{ccc}
F_{00}^{(n)} & \sqrt{\tilde{\alpha}} Y_{10}^{(n) T} & \sqrt{\tilde{\alpha}}^{-1} Y_{20}^{(n) T} \\
F_{10}^{(n)} & F_{11}^{(n)} & \sqrt{\tilde{\alpha}}^{-1} F_{21}^{(n) T} \\
F_{20}^{(n)} & \sqrt{\tilde{\alpha}} F_{21}^{(n)} & F_{22}^{(n)}
\end{array}\right], \\
X_{j 0}^{(n)} & =\bar{X}_{j 0}+\bar{\mu} E_{j 0}^{(n)}, Y_{j 0}^{(n)}=\bar{Y}_{j 0}+\bar{\mu} F_{j 0}^{(n)}, j=1,2,
\end{aligned}
$$

with

$$
\begin{aligned}
& E^{(0)}= {\left[\begin{array}{ccc}
E_{00}^{(0)} & \sqrt{\tilde{\alpha}} \bar{X}_{10}^{T} & \sqrt{\tilde{\alpha}}{ }^{-1} \bar{X}_{20}^{T} \\
E_{10}^{(0)} & E_{11}^{(0)} & \sqrt{\tilde{\alpha}} E^{-1} E_{21}^{(0) T} \\
E_{20}^{(0)} & \sqrt{\tilde{\alpha}} E_{21}^{(0)} & E_{22}^{(0)}
\end{array}\right], } \\
& F^{(0)}= {\left[\begin{array}{ccc}
F_{00}^{(0)} & \sqrt{\tilde{\alpha}} \bar{Y}_{10}^{T} & \sqrt{\tilde{\alpha}}{ }^{-1} \bar{Y}_{20}^{T} \\
F_{10}^{(0)} & F_{11}^{(0)} & \sqrt{\tilde{\alpha}}{ }^{-1} F_{21}^{(0) T} \\
F_{20}^{(0)} & \sqrt{\tilde{\alpha}} F_{21}^{(0)} & F_{22}^{(0)}
\end{array}\right], } \\
& D^{T} E^{(0)}+E^{(0) T} D-L^{T} F^{(0)}-F^{(0) T} L \\
&+\bar{Y}^{T} G_{2} \bar{Y}=0, \\
& D^{T} F^{(0)}+F^{(0) T} D-M^{T} E^{(0)}-E^{(0) T} M \\
&+\bar{X}^{T} G_{1} \bar{X}=0 .
\end{aligned}
$$

Using the iterative algorithm (15), we now give the highorder approximate Nash strategy (16).

$$
\begin{aligned}
u_{1 \mathrm{app}}^{(n)}(t) & =-R_{11}^{-1} B_{1}^{T}\left(\bar{X}+\bar{\mu} E^{(n-1)}\right) x(t) \\
& =-R_{11}^{-1} B_{1}^{T} X^{(n)} x(t), n=1, \ldots, \\
u_{2 \mathrm{app}}^{(n)}(t) & =-R_{22}^{-1} B_{2}^{T}\left(\bar{Y}+\bar{\mu} F^{(n-1)}\right) x(t) \\
& =-R_{22}^{-1} B_{2}^{T} Y^{(n)} x(t), n=1, \ldots .
\end{aligned}
$$

Theorem 2: Assume that

$$
\bar{\varepsilon}_{j}-\sigma_{j} \bar{\mu}^{n+1} \leq \varepsilon_{j} \leq \bar{\varepsilon}_{j}+\sigma_{j} \bar{\mu}^{n+1}, j=1,2 .
$$

Under Assumptions 1-3, the use of the high-order approximate strategy (16) results in $J_{i}\left(u_{i \text { app }}^{(n)}, u_{j \text { app }}^{(n)}\right)$ satisfying

$$
\begin{aligned}
& J_{i}\left(u_{i \text { app }}^{(n)}, u_{j \text { app }}^{(n)}\right)=J_{i}\left(u_{i}^{*}, u_{j}^{*}\right)+O\left(\bar{\mu}^{n+1}\right), \\
& i=1,2, n=1, \ldots,
\end{aligned}
$$

where $J_{i}\left(u_{i}^{*}, u_{j}^{*}\right), i=1,2$ are the optimal equilibrium values of the cost functions (2).

Proof: We prove only the case $i=1$. The proof of the case $i=2$ is similar. When $u_{1 \text { app }}^{(n)}$ is used, the value of the performance index is

$$
J_{1}\left(u_{1 \mathrm{app}}^{(n)}, u_{2 \mathrm{app}}^{(n)}\right)=\frac{1}{2} x(0)^{T} W_{1 e}^{(n)} x(0),
$$

where $W_{1 e}^{(n)}$ is the positive semidefinite solution of the following multiparameter algebraic Lyapunov equation (MALE)

$$
\begin{aligned}
& \left(A_{e}-S_{1 e} X_{e}^{(n)}-S_{2 e} Y_{e}^{(n)}\right)^{T} W_{1 e}^{(n)} \\
& \quad+W_{1 e}^{(n)}\left(A_{e}-S_{1 e} X_{e}^{(n)}-S_{2 e} Y_{e}^{(n)}\right) \\
& \quad+Q_{1}+X_{e}^{(n)} S_{1 e} X_{e}^{(n)}+Y_{e}^{(n)} G_{2 e}^{\mu} Y_{e}^{(n)}=0
\end{aligned}
$$

with

$$
A_{e}=\Phi_{e}^{-1} A, S_{i e}=\Phi_{e}^{-1} S_{i} \Phi_{e}^{-1}, G_{i e}^{\mu}=\Phi_{e}^{-1} G_{i}^{\mu} \Phi_{e}^{-1} .
$$

Subtracting (6a) from (20) we find that $V_{1 e}^{(n)}=W_{1 e}^{(n)}-X_{e}$ satisfies the following MALE

$$
\begin{aligned}
& \left(A_{e}-S_{1 e} X_{e}^{(n)}-S_{2 e} Y_{e}^{(n)}\right)^{T} V_{1 e}^{(n)} \\
& \quad+V_{1 e}^{(n)}\left(A_{e}-S_{1 e} X_{e}^{(n)}-S_{2 e} Y_{e}^{(n)}\right) \\
& \quad+\left(X_{e}^{(n)}-X_{e}\right) S_{1 e}\left(X_{e}^{(n)}-X_{e}\right) \\
& \quad+Y_{e} S_{2 e}\left(X_{e}^{(n)}-X_{e}\right)+\left(X_{e}^{(n)}-X_{e}\right) S_{2 e} Y_{e} \\
& \quad+Y_{e}^{(n)} G_{2 e}^{\mu} Y_{e}^{(n)}-Y_{e} G_{2 e}^{\mu} Y_{e}=0 .
\end{aligned}
$$

Using the relations $X_{e}^{(n)}-X_{e}=O\left(\bar{\mu}^{n+1}\right), Y_{e}^{(n)}-Y_{e}=$ $O\left(\bar{\mu}^{n+1}\right)$ and $\bar{\mu}-\mu=O\left(\bar{\mu}^{n+1}\right)$, we can change the form of (21) into (22)

$$
\begin{aligned}
& \left(A_{e}-S_{1 e} X_{e}^{(n)}-S_{2 e} Y_{e}^{(n)}\right)^{T} V_{1 e}^{(n)} \\
& \quad+V_{1 e}^{(n)}\left(A_{e}-S_{1 e} X_{e}^{(n)}-S_{2 e} Y_{e}^{(n)}\right)+O\left(\bar{\mu}^{n+1}\right)=0 .
\end{aligned}
$$

It is easy to verify that $V_{1 e}^{(n)}=O\left(\bar{\mu}^{n+1}\right)$ because $A_{e}-$ $S_{1 e} X_{e}^{(n)}-S_{2 e} Y_{e}^{(n)}=\Phi_{e}^{-1}[D+O(\bar{\mu})]$ is stable by using the standard Lyapunov theorem [10] for sufficiently small $\bar{\mu}$. Consequently, the equality (18) holds.

Consequently, although $\varepsilon_{j}$ is unknown, we can design the high-order $O\left(\bar{\mu}^{n+1}\right)$ approximate strategy which achieves the $O\left(\bar{\mu}^{n+1}\right)$ approximation for the equilibrium value of the cost functional.

Using the similar technique of the proof of Theorem 2, the following conditions are satisfied. 
Theorem 3: Under Assumptions 1-3, the following result holds.

$$
J_{i}\left(u_{i}, u_{j \text { app }}^{(n)}\right)=J_{i}\left(u_{i}, u_{j}^{*}\right)+O\left(\bar{\mu}^{n+1}\right) .
$$

Finally, by using the similar manner which has been established in [2], the main result is easily derived.

Theorem 4: Under Assumptions 1-3, the use of the high-order strategies (16) results in (24)

$$
J_{i}\left(u_{i \text { app }}^{(n)}, u_{j \text { app }}^{(n)}\right) \leq J_{i}\left(u_{i}, u_{j \text { app }}^{(n)}\right)+O\left(\bar{\mu}^{n+1}\right) .
$$

Proof: Let us rewrite an inequality (24) as

$$
\begin{gathered}
J_{i}\left(u_{i \text { app }}^{(n)}, u_{j \text { app }}^{(n)}\right)-J_{i}\left(u_{i}, u_{j \text { app }}^{(n)}\right) \\
=J_{i}\left(u_{i \text { app }}^{(n)}, u_{j \text { app }}^{(n)}\right)-J_{i}\left(u_{i}^{*}, u_{j}^{*}\right) \\
\quad+J_{i}\left(u_{i}^{*}, u_{j}^{*}\right)-J_{i}\left(u_{i}, u_{j}^{*}\right) \\
\quad+J_{i}\left(u_{i}, u_{j}^{*}\right)-J_{i}\left(u_{i}, u_{j \text { app }}^{(n)}\right) .
\end{gathered}
$$

Using (18), (5) and (23), the proof of (24) completes. The other case is similar.

We will present an important implication. If the parameters $\bar{\varepsilon}_{j}$ are unknown and $R_{i j}=0, i \neq j$, then the following corollary is easily seen in view of Theorem 4.

Corollary 1: Under Assumptions 1-3, the use of the parameter-independent strategies

$$
\begin{aligned}
& u_{1 \mathrm{app}}^{(0)}(t)=-R_{11}^{-1} B_{1}^{T} \bar{X} x(t), \\
& u_{2 \mathrm{app}}^{(0)}(t)=-R_{22}^{-1} B_{2}^{T} \bar{Y} x(t),
\end{aligned}
$$

results in

$$
J_{i}\left(u_{i \text { app }}^{(0)}, u_{j \text { app }}^{(0)}\right) \leq J_{i}\left(u_{i}, u_{j \text { app }}^{(0)}\right)+O(\bar{\mu}) .
$$

Proof: Since the result of Corollary 1 can be proved by using the similar technique in Theorem 4 under the fact that $X-\bar{X}=O(\bar{\mu})$ and $Y-\bar{Y}=O(\bar{\mu})$, the proof is omitted.

It is worth pointing out that the result of (27) is derived without the nonsingularity assumption of $A_{i i}$ compared with the existing one [2].

\section{NUMERICAL EXAMPLE}

In order to demonstrate the efficiency of our proposed approximate strategy, we have run a numerical example. The system matrix is given as Appendix A in [1].

$$
\begin{aligned}
& A_{00}=\left[\begin{array}{ccccc}
0 & 0 & 4.5 & 0 & 1 \\
0 & 0 & 0 & 4.5 & -1 \\
0 & 0 & -0.05 & 0 & -0.1 \\
0 & 0 & 0 & -0.05 & 0.1 \\
0 & 0 & 32.7 & -32.7 & 0
\end{array}\right], \\
& A_{01}=\left[\begin{array}{cc}
0 & 0 \\
0 & 0 \\
0.1 & 0 \\
0 & 0 \\
0 & 0
\end{array}\right], A_{02}=\left[\begin{array}{cc}
0 & 0 \\
0 & 0 \\
0 & 0 \\
0.1 & 0 \\
0 & 0
\end{array}\right] \\
& A_{10}=\left[\begin{array}{ccccc}
0 & 0 & 0 & 0 & 0 \\
0 & 0 & -0.4 & 0 & 0
\end{array}\right] \text {, }
\end{aligned}
$$

$$
\begin{aligned}
& A_{20}=\left[\begin{array}{ccccc}
0 & 0 & 0 & 0 & 0 \\
0 & 0 & 0 & -0.4 & 0
\end{array}\right] \text {, } \\
& A_{11}=A_{22}=\left[\begin{array}{cc}
-0.05 & 0.05 \\
0 & -0.1
\end{array}\right] \text {, } \\
& B_{01}=B_{02}=\left[\begin{array}{lllll}
0 & 0 & 0 & 0 & 0
\end{array}\right]^{T} \text {, } \\
& B_{11}=B_{22}=\left[\begin{array}{c}
0 \\
0.1
\end{array}\right] \text {, } \\
& R_{11}=R_{22}=20, R_{12}=R_{21}=0 \text {, } \\
& Q_{1}=\operatorname{diag}\left(\begin{array}{lllllllll}
1 & 1 & 1 & 1 & 1 & 1 & 1 & 0 & 0
\end{array}\right) \text {, } \\
& Q_{2}=\operatorname{diag}\left(\begin{array}{lllllllll}
1 & 1 & 1 & 1 & 1 & 0 & 0 & 1 & 1
\end{array}\right) \text {. }
\end{aligned}
$$

It is assumed that the parameters $\varepsilon_{j}$ satisfy the following constraint.

$$
\bar{\varepsilon}_{j}-\sigma_{j} \bar{\mu}^{2} \leq \varepsilon_{j} \leq \bar{\varepsilon}_{j}+\sigma_{j} \bar{\mu}^{2}, j=1,2 .
$$

Therefore, the desired approximation on the basis of the iterative algorithm for the construction of high-order strategy is up to one, that is, $n=1$. Using the results of this paper the solutions $\bar{X}$ and $\bar{Y}$ of (11) are given in Table1. We evaluate the costs using the approximate Nash strategies $u_{j \text { app }}^{(0)}$ which is given by (26) and the proposed high-order Nash strategies $u_{j \text { app }}^{(1)}$. We assume that the initial conditions are zero mean independent random vector with covariance matrix.

$$
\begin{aligned}
& E\left[x(0) x(0)^{T}\right] \\
= & 10^{-4} \operatorname{diag}(1,1,0.01,0.01,1,1,1,1,1)(32)
\end{aligned}
$$

Moreover, let us define the error equations between the optimal cost and the approximate cost.

$$
\begin{aligned}
& \delta_{j}^{(n)}:=\frac{\left|E\left[J_{i}\left(u_{i \mathrm{app}}^{(n)}, u_{j \mathrm{app}}^{(n)}\right)\right]-E\left[J_{i}\left(u_{i}^{*}, u_{j}^{*}\right)\right]\right|}{\bar{\mu}^{n+1}}, \\
& j=1,2, n=0,1 .
\end{aligned}
$$

The average value of the cost functionals $J_{i}\left(u_{i}^{*}, u_{j}^{*}\right)$, $J_{i}\left(u_{i \text { app }}^{(n)}, u_{j \text { app }}^{(n)}\right)$ and the errors $\delta_{j}^{(n)}$ are given in Table 2 and Table 3 . These show that $u_{j \text { app }}^{(1)} x(t)$ has improved the cost functional $J_{i}\left(u_{i \text { app }}^{(0)}, u_{j \text { app }}^{(0)}\right)$. Finally, even if the small perturbation parameters are unknown, the proposed high-order approximate strategies guarantee the desired performance level as long as the inequality (31) holds.

\section{CONCLUSION}

The linear quadratic Nash games for infinite horizon nonstandard MSPS have been studied. Firstly, the uniqueness, the boundedness and the asymptotic structure of the solution for the GCMARE have been newly proved. Secondly, the high-order approximate strategy which is based on the new iterative algorithm has been proposed without the exact information of the singular perturbation parameters. As a result, for values of $\mu$ that are not too small, the proposed higher order approximations strategies attain the desired performance. Moreover, since the nonsingularity assumptions for the fast state matrices are not needed, our 
Table 1.

$$
\begin{aligned}
& \bar{X}_{00}=\left[\begin{array}{rrrrr}
5.5643 & 7.7043 e-1 & 4.6904 e+1 & -2.9538 e-2 & 2.4629 e-1 \\
7.7043 e-1 & 3.2860 & 1.9692 e-2 & 2.3452 e+1 & -3.1520 e-1 \\
4.6904 e+1 & 1.9692 e-2 & 6.4215 e+2 & -2.1432 e+2 & 4.7125 \\
-2.9538 e-2 & 2.3452 e+1 & -2.1432 e+2 & 4.0104 e+2 & -5.7292 \\
2.4629 e-1 & -3.1520 e-1 & 4.7125 & -5.7292 & 1.2843
\end{array}\right] \\
& \bar{X}_{10}= {\left[\begin{array}{rrrrr}
9.2357 e+1 & 3.8775 e-2 & 1.2397 e+3 & -4.2201 e+2 & 9.2791 \\
4.4721 e+1 & 1.8776 e-2 & 5.7503 e+2 & -2.0435 e+2 & 4.4932
\end{array}\right] } \\
& \bar{X}_{20}=\left[\begin{array}{rrrrr}
-5.8162 e-2 & 4.6178 e+1 & -4.2201 e+2 & 7.8967 e+2 & -1.1281 e+1 \\
-2.8163 e-2 & 2.2361 e+1 & -2.0435 e+2 & 3.8237 e+2 & -5.4626
\end{array}\right], \bar{X}_{11}=\left[\begin{array}{lrr}
9.9473 & 3.2453 \\
3.2453 & 6.5165
\end{array}\right] \\
& \bar{Y}_{00}=\left[\begin{array}{rrrrr}
3.2860 & 7.7043 e-1 & 2.3452 e+1 & 1.9692 e-2 & 3.1520 e-1 \\
7.7043 e-1 & 5.5643 & -2.9538 e-2 & 4.6904 e+1 & -2.4629 e-1 \\
2.3452 e+1 & -2.9538 e-2 & 4.0104 e+2 & -2.1432 e+2 & 5.7292 \\
1.9692 e-2 & 4.6904 e+1 & -2.1432 e+2 & 6.4215 e+2 & -4.7125 \\
3.1520 e-1 & -2.4629 e-1 & 5.7292 & -4.7125 & 1.2843
\end{array}\right] \\
& \bar{Y}_{10}=\left[\begin{array}{llrrr}
4.6178 e+1 & -5.8162 e-2 & 7.8967 e+2 & -4.2201 e+2 & 1.1281 e+1 \\
2.2361 e+1 & -2.8163 e-2 & 3.8237 e+2 & -2.0435 e+2 & 5.4626
\end{array}\right] \\
& \bar{Y}_{20}=\left[\begin{array}{lllll}
3.8775 e-2 & 9.2357 e+1 & -4.2201 e+2 & 1.2397 e+3 & -9.2791 \\
1.8776 e-2 & 4.4721 e+1 & -2.0435 e+2 & 5.7503 e+2 & -4.4932
\end{array}\right], \bar{Y}_{22}=\left[\begin{array}{rr}
9.9473 & 3.2453 \\
3.2453 & 6.5165
\end{array}\right]
\end{aligned}
$$

\begin{tabular}{|c|c|c|c|c|c|c|c|}
\hline$\varepsilon_{1}$ & $\varepsilon_{2}$ & $J_{1}\left(u_{1 \mathrm{app}}^{(0)}, u_{2 \mathrm{app}}^{(0)}\right.$ & $J_{2}\left(u_{2 \mathrm{app}}^{(0)}, u_{2 \mathrm{app}}^{(0)}\right)$ & $J_{1}\left(u_{1}^{*}, u_{2}^{*}\right)$ & $J_{2}\left(u_{1}^{*}, u_{2}^{*}\right)$ & $\delta_{1}^{(0)}$ & $\delta_{2}^{(0)}$ \\
\hline $10^{-2}$ & $10^{-2}$ & $1.2637 e-3$ & $1.2637 e-3$ & $1.2314 e-3$ & $1.2314 e-3$ & $3.2317 e-3$ & $3.2317 e-3$ \\
\hline $10^{-2}$ & $5 \times 10^{-3}$ & $1.1773 e-3$ & $1.1602 e-3$ & $1.1611 e-3$ & $1.1644 e-3$ & $2.2959 e-3$ & $5.9933 e-4$ \\
\hline $10^{-3}$ & $10^{-3}$ & $1.0356 e-3$ & $1.0356 e-3$ & $1.0388 e-3$ & $1.0388 e-3$ & $3.2315 e-3$ & $3.2315 e-3$ \\
\hline $10^{-3}$ & $5 \times 10^{-4}$ & $1.0344 e-3$ & $1.0329 e-3$ & $1.0361 e-3$ & $1.0361 e-3$ & $2.4272 e-3$ & $4.5428 e-3$ \\
\hline $10^{-4}$ & $10^{-4}$ & $1.0289 e-3$ & $1.0289 e-3$ & $1.0293 e-3$ & $1.0293 e-3$ & $3.5009 e-3$ & $3.5009 e-3$ \\
\hline \multicolumn{8}{|c|}{ Table 3.} \\
\hline$\varepsilon_{1}$ & $\varepsilon_{2}$ & $J_{1}\left(u_{1 \mathrm{app}}^{(1)}, u_{2 \mathrm{app}}^{(1)}\right.$ & $J_{2}\left(u_{2 \mathrm{app}}^{(1)}, u_{2 \mathrm{app}}^{(1)}\right)$ & $J_{1}\left(u_{1}^{*}, u_{2}^{*}\right)$ & $J_{2}\left(u_{1}^{*}, u_{2}^{*}\right)$ & $\delta_{1}^{(1)}$ & $\delta_{2}^{(1)}$ \\
\hline $10^{-2}$ & $10^{-2}$ & $1.2252 e-3$ & $1.2252 e-3$ & $1.2314 e-3$ & $1.2314 e-3$ & $6.1218 e-2$ & $6.1218 e-2$ \\
\hline $10^{-2}$ & $5 \times 10^{-3}$ & $1.1665 e-3$ & $1.1539 e-3$ & $1.1611 e-3$ & $1.1644 e-3$ & $1.0903 e-1$ & $2.1043 e-1$ \\
\hline $10^{-3}$ & $10^{-3}$ & $1.0388 e-3$ & $1.0388 e-3$ & $1.0388 e-3$ & $1.0388 e-3$ & $5.6898 e-2$ & $5.6898 e-2$ \\
\hline $10^{-3}$ & $5 \times 10^{-4}$ & $1.0361 e-3$ & $1.0360 e-3$ & $1.0361 e-3$ & $1.0361 e-3$ & $6.3971 e-2$ & $2.0829 e-1$ \\
\hline $10^{-4}$ & $10^{-4}$ & $1.0293 e-3$ & $1.0293 e-3$ & $1.0293 e-3$ & $1.0293 e-3$ & $5.6335 e-2$ & $5.6335 e-2$ \\
\hline
\end{tabular}

Table 2.

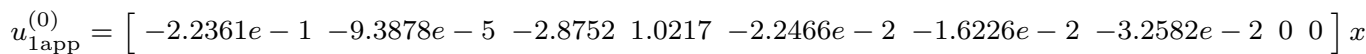

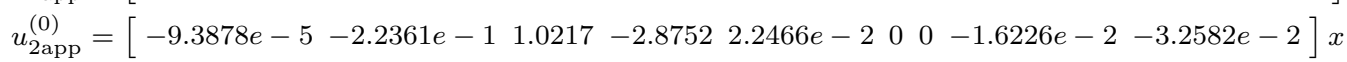

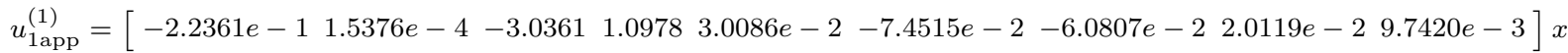

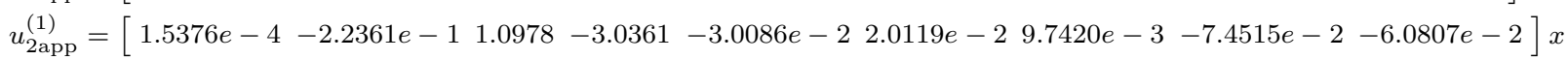

$u_{1}^{*}(t)=\left[\begin{array}{lllll}-2.2361 e-1 & 2.0084 e-4-2.9106 & 9.7258 e-1 & 3.1611 e-2-7.5473 e-2-6.0799 e-2 & 2.0153 e-29.4638 e-3\end{array}\right] x$

$u_{2}^{*}(t)=\left[\begin{array}{llll}2.0084 e-4-2.2361 e-1 & 9.7258 e-1-2.9106-3.1611 e-2 & 2.0153 e-29.4638 e-3-7.5473 e-2-6.0799 e-2\end{array}\right] x(30 \mathrm{~b})$

proposed method is applicable to wider class of the practical MSPS.

\section{REFERENCES}

[1] H. K. Khalil and P. V. Kokotović, "Control strategies for decision makers using different models of the same system," IEEE Trans. Automatic Control, vol. 23, no.4, pp. 289-298, 1978.

[2] H. K. Khalil, "Multimodel design of a Nash strategy," Journal of Optimization Theory and Applications, vol. 31, no. 4, pp.553-564, 1980.

[3] H. K. Khalil and P. V. Kokotovic, "Control of linear systems with multiparameter singular perturbations," Automatica, vol. 15, no. 2, pp.197-207, 1979.

[4] S. Koskie, D. Skataric and B. Petrovic, "Convergence proof for recursive solution of linear-quadratic Nash games for quasi-singularly perturbed systems," Dynamics Continuous, Discrete and Impulsive Systems, vol.9b, pp.317-333, 2002.

[5] T-Y. Li and Z. Gajić, Lyapunov Iterations for Solving Coupled Algebraic Lyapunov Equations of Nash Differential Games and Algebraic Riccati Equations of Zero-Sum Games, New Trends in Dynamic Games and Applications, Boston: Birkhauser, pp.333-351, 1994.
[6] M. T. Lim, C.-G. Kang and B.-S. Kim, "Optimal control of linear nonstandard singularly perturbed discrete systems," Dynamics Continuous, Discrete and Impulsive Systems, vol. 9, no. 2, pp.163-174, 2002.

[7] H. Mukaidani, H. Xu and K. Mizukami, Numerical Algorithm for Solving Cross-Coupled Algebraic Riccati Equations of Singularly Perturbed Systems, Annals of Dynamic Games, (to appear)

[8] H. Mukaidani, T, Shimomura and H. Xu, Numerical algorithm for solving cross-coupled algebraic Riccati equations related to Nash games of multimodeling systems, In proceedings of the 41th IEEE Conf. Decision and Control, pp.4167-4172, 2002.

[9] H. Mukaidani, T, Shimomura and H. Xu, Asymptotic expansions of solutions of cross-coupled algebraic Riccati equations of multimodeling systems related to Nash Games, In proceedings of the 3rd International DCDIS Conference on Engineering Applications and Computational Algorithms, pp.34-39, 2003.

[10] K. Zhou, Essentials of Robust Control, Prentice-Hall, New Jersey: 1998. 